Article

\title{
A Guaranteed Deterministic Approach to Superhedging-The Case of Convex Payoff Functions on Options
}

\author{
Sergey Smirnov ${ }^{1,2}$ \\ 1 Faculty of Computational Mathematics and Cybernetics, Lomonosov Moscow State University, Leninskie \\ Gory 1/52, 119991 Moscow, Russia; s.n.smirnov@gmail.com \\ 2 Financial Engineering and Risk Management Laboratory, National Research University Higher School of \\ Economics, 20 Myasnitskaya Ulitsa, 101000 Moscow, Russia
}

Received: 29 July 2019; Accepted: 12 December 2019; Published: 17 December 2019; Corrected: 30 November 2022

check for updates

\begin{abstract}
This paper considers super-replication in a guaranteed deterministic problem setting with discrete time. The aim of hedging a contingent claim is to ensure the coverage of possible payoffs under the option contract for all admissible scenarios. These scenarios are given by means of a priori given compacts that depend on the history of prices. The increments of the price at each moment in time must lie in the corresponding compacts. The absence of transaction costs is assumed. The game-theoretic interpretation of pricing American options implies that the corresponding Bellman-Isaacs equations hold for both pure and mixed strategies. In the present paper, we study some properties of the least favorable (for the "hedger") mixed strategies of the "market" and of their supports in the special case of convex payoff functions.
\end{abstract}

Keywords: guaranteed estimates; deterministic price dynamics; super-replication; option; no arbitrage condition; Bellman-Isaacs equations; multi-valued mapping; semi-continuity; mixed strategies; game equilibrium; convex payoff functions

MSC: 91A25; 91G20

\section{JEL Classification: $\mathrm{C} 6 ; \mathrm{C} 7$}

\section{Introduction}

The present article continues the series of publications by the author [1-8]. These works develop a model of the financial market with an uncertain deterministic evolution of prices and discrete time. Asset prices evolve in a deterministic manner under the uncertainty described by a priori information on possible price increments. It is assumed that they lie in given compact sets depending on the history of prices. This model is an alternative to traditional probabilistic market models (The deterministic approach that we propose does not need a reference probabilistic measure, as is assumed within the probabilistic approach. A modern exposition of the probabilistic approach can be found in Reference [9].). A number of authors, particularly Fölmer and Shid [9], qualify alternative (to probabilistic) problem statements as formalizations of the Knightian uncertainty. This qualification, however, seems to us incorrect, since Knight [10] (chapter 8) speaks of uncertainty that cannot be quantified (as opposed to risk). In our works, we use the term "guaranteed deterministic approach". This approach is applicable both for the problem of pricing and hedging options and for the problem of portfolio investment (see Reference [11]).

In the aforementioned series of publications, we focused on the issue of superhedging options in the presence of trade constraints. (The superhedging pricing of an option determines the minimum 
level of funds at the initial moment necessary for the seller of an option to guarantee coverage of the contingent liability (the potential payouts that depend on the history of prices) by choosing an appropriate (admissible) hedging strategy. Note that a wide range of pricing problems concerning contingent claims can be reduced to the superhedging pricing for the modified payoff structure (see Reference [9], Chapter 8).) In general, we considered American options; European and Bermuda options can be considered particular cases of American options under an economically natural assumption of "no arbitrage" in the market (in a certain sense). Note that the above-mentioned assumption about the compactness of the set of possible values of price increments (recall that these compacts depend on the history of prices) is essential for the problem of superhedging to be meaningful, since, under the unlimited support of conditional distributions of price increments and a given price history, one should generally expect degenerate hedging results, such as those described in Reference [12]. This is the reason why the authors of Reference [13] assume limited increments in the super-replication problem (albeit in a probabilistic setting) with one risky asset.

One of the first publications to develop a guaranteed deterministic approach is an article by Kolokoltsov [14], published in 1998. To our best knowledge, this is the first work to explicitly articulate this approach to pricing and hedging contingent option obligations. Implicitly, however, some mathematical tools for a guaranteed deterministic approach were already present in 1994 ([15] Sections 1.1.6 and 1.2.4). The result of the first part of Reference [14] (the case of a single risky asset and a convex payout function on European option) directly follows from Reference [15].

The guaranteed deterministic approach is closely related to a class of market models called interval models in Reference [16]. Our model and results are especially close to the ideas and results of V.N. Kolokoltsov (Chapters 11-14 of Reference [16]), including independent discovery of the game-theoretic interpretation of risk-neutral probabilities (we find this important from an economic point of view. In Reference [17], we present our thoughts on the importance of the meaningful interpretation of the concepts, assumptions, and mathematical results of financial models. We note that the perception of the risk-neutral valuation of financial instruments is of fundamental importance for the correct understanding of the phenomenon of "bubbles" in financial markets) under the assumption of no trading constraints; we find this interpretation to be quite important from an economic point of view. In Chapters 13 and 14 of Reference [16], dealing with the problem of pricing European and American options, the restrictions imposed on the logarithms of prices are assumed to be intervals, that is, the vectors of the logarithms of price increments lie in the parallelepiped, although the theory presented in Chapter 12 [16] allows one, in principle, to consider compact-valued restrictions on price movements, as in our work. The issues of game equilibrium in mixed strategies with trade restrictions and the role of "no arbitrage" assumptions for game equilibrium are not addressed in Reference [16]. Also, the relationship between deterministic and stochastic approaches is not discussed, though the solutions for these approaches may differ in general ( the model considered in Chapters 13 and 14 of Reference [16] corresponds to the fairly regular (Lipschitzian) behavior of multi-valued mappings, so Kolokoltsov's deterministic model, as a rule, produces the same results as the stochastic approach. The answer to this question, however, also depends on the "smoothness" of payment functions. Therefore, a mismatch between the results of the two approaches may take place even within the framework of Kolokoltsov's model (for example, for binary options)).

Over the past few years, there has been increasing interest in a new approach to modeling uncertainty in the market called a "robust" or (less successfully) "model independent" method. A guaranteed deterministic approach can clearly be considered as robust. The works related to a robust approach can be divided into two directions: a quasi-sure approach and a pathwise approach. In related studies, two categories of financial instruments are usually distinguished in a portfolio. The first is trading, consisting of instruments traded dynamically (underlying assets) and the second is static, based on buy and hold strategies, usually using European call and put options with the same time of execution. The idea of studying these kinds of portfolios belongs to Hobson [18]. The relationship of pathwise and quasi-sure approaches was established in Reference [19]. 
This quasi-sure approach introduces a class of probabilistic measures that describe possible scenarios of market behavior. In contrast to the traditional probabilistic approach, this class can contain mutually singular measures, which create serious mathematical complications. The idea of a model with discrete time was introduced in Reference [20]. The choice of a class of probabilistic measures has produced a wide range of specifications for market dynamics. This approach has been used to describe market uncertainty in a number of papers (for example, References [21,22]).

The pathwise approach to describing the uncertainty in market modeling is defined through specific market scenarios, without the use of probabilistic measures. The general theory of the trajectory approach for a discrete-time model was constructed in Reference [23] based on the earlier developments in References [24,25]. Kolokoltsov's work in Reference [16] and our study can be formally considered pathwise types. However, they differ in their use of "local in time" restrictions on possible trajectories, which leads to simpler economic interpretations and convenient use in practice. Also, the static part of the portfolio of financial instruments is not considered in these models.

In Reference [26], the research started in References [20,25] was continued. For a discrete time model, some general results on the duality of pricing have been obtained for American options. The authors managed to successfully apply dynamic principle programming via fictitious market extension, where all financial instruments are traded dynamically. Note that our approach is also based on the use of the principle of dynamic programming.

Below we provide a description of the concepts necessary for the purposes of this paper.

The key premise of the proposed approach is the formulation of "uncertain" price dynamics in terms of information on price movements (the increments are taken "backward", that is, $\Delta X_{t}=$ $X_{t}-X_{t-1}$, where $X_{t}$ is the discounted price vector at time $t$ and the $i$-th component of this vector is the per unit price of the $i$-th asset. ) observed prior to time $t$. Specifically, possible increments $\Delta X_{t}$ of discounted prices ( we suppose that a risk-free asset has a fixed price equal to one) belong to a priori given compacts $K_{t}(\cdot) \subseteq \mathbb{R}^{n}$, where the dot indicates the price prehistory known up to time $t-1$, $t=1, \ldots, N$. Let $v_{t}^{*}(\cdot)$ denote the infimum of portfolio value at time $t$ that guarantees coverage of current and future possible claims on the American option given the price history and appropriately chosen hedging strategy. The corresponding Bellman-Isaacs equations expressed in discounted prices naturally arise from economic principals and are determined by selecting at step $t$ the "best" admissible hedging strategy ( the vector $h$ describes the size of positions held in assets, i.e, the $i$-th component of this vector is the number of units of the $i$-th asset purchased or sold.) $h \in D_{t}(\cdot) \subseteq \mathbb{R}^{n}$ for the "worst" scenario of (discounted) price movements $y \in K_{t}(\cdot)$ for given potential option payoff functions $g_{t}(\cdot)$.

Let us first present a simple derivation of master equations, at the "engineering" level of rigor, by applying the principle of dynamic programming. Assume for simplicity that the supremum and infimum in (1) are attained. Then, the economic meaning of theBellman-Isaacs equations can be explained in the following (although slightly informal) way. Let $t \leq N$ and $x_{1}, \ldots, x_{t-1}$ be a history of price dynamics known up to time $t-1$, which is considered the present. To meet contingent liabilities on sold American options for certain, first, the value $V_{t-1}$ of the hedging portfolio should be no less than current liabilities $g_{t}\left(x_{1}, \ldots, x_{t-1}\right)$ and, second, the value of the hedging portfolio in the next period $V_{t}=V_{t-1}+H_{t} \Delta X_{t}$ (the strategy $H_{t}$ is selected at time $t-1$ and determined only by the price history $\left.x_{1}, \ldots, x_{t-1}\right)$ should be no less than $v_{t}^{*}\left(x_{1}, \ldots, x_{t-1}, x_{t-1}+y\right)$ for any possible scenario for one-step price movement $\Delta X_{t}=y \in K_{t}\left(x_{1}, \ldots, x_{t-1}\right)$. Therefore, when choosing a strategy $H_{t}=h \in$ $D_{t}\left(x_{1}, \ldots, x_{t-1}\right)$ one must ensure that the portfolio value $V_{t-1}$ is at least $v_{t}^{*}\left(x_{1}, \ldots, x_{t-1}, x_{t-1}+y\right)-h y$ in the most unfavorable price movement scenario $y \in K_{t}\left(x_{1}, \ldots, x_{t-1}\right)$ at step $t$, that is, in scenario $y \in K_{t}\left(x_{1}, \ldots, x_{t-1}\right)$ that maximizes the expression $v_{t}^{*}\left(x_{1}, \ldots, x_{t-1}, x_{t-1}+y\right)-h y$. By minimizing this value by a properly selected strategy $h \in D_{t}\left(x_{1}, \ldots, x_{t-1}\right)$, one can estimate the minimum reserves required to meet any possible future payment on the option. Since the optimal hedging portfolio covers current and future claims on the option, its value $v_{t}^{*}\left(x_{1}, \ldots, x_{t-1}\right)$ is supposed to be equal to the maximum of two-current liabilities and minimum reserves for covering future payments. 
Thus, formalizing the construction described above, we obtain the following recurrence relations: ( The notation $\vee$ indicates the maximum, and $h y=\langle h, y\rangle$ is the vector dot product $h$ to vector $y$.)

$$
\begin{gathered}
v_{N}^{*}\left(\bar{x}_{N}\right)=g_{N}\left(\bar{x}_{N}\right), \\
v_{t-1}^{*}\left(\bar{x}_{t-1}\right)=g_{t-1}\left(\bar{x}_{t-1}\right) \bigvee \inf _{h \in D_{t}\left(\bar{x}_{t-1}\right)} \sup _{y \in K_{t}\left(\bar{x}_{t-1}\right)}\left[v_{t}^{*}\left(\bar{x}_{t-1}, x_{t-1}+y\right)-h y\right], \\
\quad t=N, \ldots, 1,
\end{gathered}
$$

where $\bar{x}_{t-1}=\left(x_{0}, \ldots, x_{t-1}\right)$ presents the price history up to the current moment $t$. The conditions for the validity of (1) are formulated in Theorem 3.1 in [1].

Assume for convenience that $g_{0}=-\infty$, that is, there is no claim on the option at the initial moment; $g_{t} \geq 0$ for $t=1, \ldots, N$ for an American option. The sets $D_{t}(\cdot)$ are assumed to be convex and $0 \in D_{t}(\cdot)$.

Multi-valued mappings $x \mapsto K_{t}(x)$ and $x \mapsto D_{t}(x)$, as well as functions $x \mapsto g_{t}(x)$, are assumed to be defined for all $x \in\left(\mathbb{R}^{n}\right)^{t}, t=1, \ldots, N$. Therefore, the functions $x \mapsto v_{t}^{*}(x)$ are defined by Equation (1) for all $x \in\left(\mathbb{R}^{n}\right)^{t}$.

In Equation (1), the functions $v_{t}^{*}$, as well as the corresponding supremum and infimum, take values in an extended set of real numbers $\mathbb{R} \cup\{-\infty,+\infty\}=(-\infty,+\infty)$, that is, a two-point compactification (the neighborhoods of points $-\infty$ and $+\infty$ are given by $[\infty, a), a \in \mathbb{R}$ and $(b,+\infty), b \in \mathbb{R}$, respectively.) of $\mathbb{R}$.

Let us consider an asset price trajectory $\left(x_{0}, \ldots, x_{t}\right)=\bar{x}_{t}$ over the period $[0, t]=\{0, \ldots, t\}$ as being possible if $x_{0} \in K_{0}, \Delta x_{1} \in K_{1}\left(x_{0}\right), \ldots, \Delta x_{t} \in K_{t}\left(x_{0}, \ldots, x_{t-1}\right)$, where $t=0,1, \ldots, N$ and denote by $B_{t}$ the sets of all possible trajectories of asset prices over the period $[0, t]$ :

$$
B_{t}=\left\{\left(x_{0}, \ldots, x_{t}\right): x_{0} \in K_{0}, \Delta x_{1} \in K_{1}\left(x_{0}\right), \ldots, \Delta x_{t} \in K_{t}\left(x_{0}, \ldots, x_{t-1}\right)\right\} .
$$

Note that one of the important conditions for the validity of (1), formulated in Theorem 3.1 in Reference [1], is the boundedness of payoff functions $g_{t}$, which ensures that functions $v_{t}^{*}$ are bounded from above:

There are constants $C_{t} \geq 0$ such that for every $t=1, \ldots, N$ and any possible price evolution $\bar{x}_{t}=\left(x_{0}, \ldots, x_{t}\right) \in B_{t}$ the inequality $g_{t}\left(x_{0}, \ldots, x_{t}\right) \leq C_{t}$ holds.

Henceforth, we replace the last variable in functions $v_{t}^{*}$ with its "additive" representation for the convenience of notation:

$$
w\left(\bar{x}_{t-1}, y\right)=w_{t}\left(x_{1}, \ldots, x_{t-1}, y\right)=v^{*}\left(x_{1}, \ldots, x_{t-1}, x_{t-1}+y\right),
$$

and we can write the Bellman-Isaacs equations in terms of the functions $w_{t}, t=N, \ldots, 0$ as follows:

$$
v_{t-1}^{*}(\cdot)=g_{t-1}(\cdot) \bigvee \inf _{h \in D_{t}(\cdot)} \sup _{y \in K_{t}(\cdot)}\left[w_{t}(\cdot, y)-h y\right], \quad t=N, \ldots, 1 .
$$

Let us now sketch the content of the above-mentioned series of our papers.

In Reference [1], we describe in detail the guaranteed deterministic approach, refer to the relevant literature, and formulate a corresponding financial market model with trading constraints. We also frame the problem of superhedging contingent claims on options, obtain the Bellman-Isaacs equations (1), and discuss what kinds of economic assumptions are needed to ensure the equation's validity. Below we admit all the assumptions listed in Theorem 3.1 in Reference [1] and those listed in Item 1) of Remark 3.1 in Reference [1].

In Reference [2], different notions of "no-arbitrage" such as no sure arbitrage, no arbitrage opportunities, and no sure arbitrage with unbounded profit are introduced and studied in a 
deterministic market model. The paper also introduces the concept of the robustness of the "no-arbitrage" property (structural stability of the model) and obtains the criteria for robustness.

For the convenience of the reader we give several definitions of the notions introduced in Reference [2].

By the (deterministic) arbitrage opportunity (AO) at the time step $t$ we mean the following:

1. there exists a strategy $h^{*} \in D_{t}(\cdot)$, such that $h^{*} y \geq 0$ for all $y \in K_{t}(\cdot)$,

2. there exists a price movement $y^{*} \in K_{t}(\cdot)$, such that $h^{*} y^{*}>0$.

By the (deterministic) sure arbitrage (SA) at the time step $\mathrm{t}$ we mean the following: there exists a strategy $h^{*} \in D_{t}(\cdot)$ such that $h^{*} y>0$ for all $y \in K_{t}(\cdot)$.

We say that there is a (deterministic) sure arbitrage with unlimited profit (SAUP) at the time step $t$ if the function (It can be interpreted as the guaranteed profit for a strategy $h \in D_{t}(\cdot)$. Note that $\min \left\{h y, y \in K_{t}(\cdot)\right\}$ is attained due to the compactness of $K_{t}(\cdot)$ for some (worst scenario) $y_{h}^{*} \in K_{t}(\cdot)$; it is positive for the sure arbitrage strategy $\left.h^{*} \in D_{t}(\cdot).\right) h \mapsto \min \left\{h y, y \in K_{t}(\cdot)\right\}$ takes arbitrarily large values for $h \in D_{t}(\cdot)$.

Using these three notions of "arbitrage" we can define the corresponding "no arbitrage" properties on a time interval; any "no arbitrage" propriety on a time interval tantamount to "no arbitrage" propriety at every time step of this interval. We get so the following "no arbitrage" properties:

1. no deterministic arbitrage opportunity (NDAO),

2. no deterministic sure arbitrage (NDSA),

3. deterministic sure arbitrage with unlimited profit (NDSAUP).

The structural stability in our context means that the qualitative behavior of a price dynamics $K_{t}(\cdot)$, namely some "no arbitrage" property, is unaffected by sufficiently small (with respect to the Pompeiu-Hausdorff metric) perturbations of $K_{t}(\cdot)$; such a "no arbitrage" property is called robust. In what follows we need two robust "no arbitrage" properties:

- robust no deterministic arbitrage opportunity (RNDAO),

- robust deterministic sure arbitrage with unlimited profit (RNDSAUP).

Reference [3] investigates the properties of the semicontinuity and continuity of solutions of the Bellman-Isaacs equations (1). The main result of that paper relates to the "smoothness" of solutions (1) (see [3], Theorem 3.2 oncontinuity $\left.v_{t}^{*}(\cdot), t=0, \ldots, N\right)$ and is obtained under the rather weak assumption of "no-arbitrage", RNDSAUP.

Proposition 2.1 in Reference [3] provides a sufficient condition for the compactness of $B_{t}$, namely, the upper semicontinuity of compact-valued mappings $x \mapsto K_{t}(\cdot), t=1, \ldots, N$, and a sufficient condition for the validity of property (3), namely, the upper semicontinuity of $K_{t}(\cdot)$ and the upper semicontinuity of $g_{t}(\cdot), t=1, \ldots, N$.

Under the conditions in Theorem 3.2 from Reference [3] and no trading constraints, in Reference [4], we estimate the modulus of the continuity of Bellman-Isaacs functions, in particular, in the case of Lipschitz functions. Reference [5] introduces the mixed extension of pure strategies of the "market" and studies the existence of game equilibrium and its properties. We use some of these results in this paper.

The justification of the transition from pure market strategies to their mixed extension is provided in Lemma 2.1 of Reference [5]. Following Reference [5], denote

$$
\begin{aligned}
& \rho_{t}(\cdot)=\inf _{h \in D_{t}(\cdot)} \sup _{Q \in \mathcal{P}_{t}(\cdot)} \int\left[w_{t}\left(\bar{x}_{t-1}, y\right)-h y\right] Q(d y), \\
& \rho_{t}^{\prime}(\cdot)=\sup _{Q \in \mathcal{P}_{t}(\cdot)} \inf _{h \in D_{t}(\cdot)} \int\left[w_{t}\left(\bar{x}_{t-1}, y\right)-h y\right] Q(d y),
\end{aligned}
$$

and assume that integrals in (5) and (6) are well defined with respect to the measure $Q$. 
The expression for $\rho_{t}(\cdot)$ appears in the Bellman-Isaacs equations for mixed strategies; following Reference [5], we can rewrite them in reduced form:

$$
\begin{gathered}
v_{N}^{*}(\cdot)=g_{N}(\cdot), \\
v_{t-1}^{*}(\cdot)=g_{t-1}(\cdot) \vee \rho_{t}(\cdot), \text { for } t=1, \ldots, N .
\end{gathered}
$$

Proposition 3.1 from Reference [5] states that if functions $w_{t}$ and a class of measures $\mathcal{P}_{t}(\cdot)$ such that integrals $\int w_{t}(\cdot, y) Q(d y)$ are defined (to avoid additional requirements on the functions $v_{t}^{*}$, one can use, for example, the class $\mathcal{P}^{*}\left(K_{t}(\cdot)\right)$ of measures concentrated in a finite set of points from $K_{t}(\cdot)$ as the $\mathcal{P}_{t}(\cdot)$. On the other hand, one can require the $v_{t}^{*}$ to be universally measurable (or have some smoothness property, for example, semicontinuity).) for any measure $Q \in \mathcal{P}_{t}(\cdot)$, then

$$
\rho_{t}^{\prime}(\cdot)=\sup _{Q \in \mathcal{P}_{t}(\cdot)}\left[\int w_{t}(\cdot, y) Q(d y)-\sigma_{D_{t}(\cdot)}\left(\int y Q(d y)\right)\right],
$$

where $\sigma_{A}$ is the support function of the set $A$.

Note that $\rho_{t}(\cdot)>-\infty$ if and only if the condition of no sure arbitrage with unbounded profit NDSAUP is fulfilled (see Remark 3.2, Item 1) in Reference [5]). If the condition of no sure arbitrage NDSA is fulfilled, then $\rho_{t}^{\prime}(\cdot) \geq 0$ (see Remark 3.5, Item 3) of Reference [5]). The inequality $\rho_{t}(\cdot) \geq \rho_{t}^{\prime}(\cdot)$ always holds; the equality $\rho_{t}(\cdot)=\rho_{t}^{\prime}(\cdot)$ represents the game equilibrium.

Item 5 of Remark 3.2 in Reference [5] shows that when there are no trading constraints, that is, $D_{t}(\cdot) \equiv \mathbb{R}^{n}$, the expression (8) takes a rather simple form (the supremum is taken, therefore, over the class of distributions, such that (discounted) prices of each risky asset are martingales.):

$$
\rho_{t}^{\prime}(\cdot)=\sup _{Q \in \mathcal{P}_{t}(\cdot), \int y Q(d y)=0} \int w_{t}(\cdot, y) Q(d y)
$$

Reference [6] studies the game equilibrium in the market with no trading constraints and no arbitrage opportunities. Its main result is represented by Theorem 1, generalizing Theorem 12.21 in Reference [16], proved by V.N. Kolokoltsov. Reference [7] proposes a two-step method for solving the Bellman-Isaacs equations in the equilibrium case (for the equilibrium existence conditions, see References $[5,6])$. The paper shows that the most unfavorable mixed market strategies can be found in the class of distributions concentrated in at most $n+1$ points, where $n$ is the number of risky assets. The paper also connects this method with the Generalized Problem of Moments; this method can be applied to other problems of hedging contingent claims. Reference [8] obtains conditions for the upper semicontinuity of multi-valued mappings, which associate a class of optimal mixed strategies of the "market" to an asset price trajectories. The paper also provides sufficient conditions for the existence of continuous selections of the supports of optimal "market" strategies that contain at most $n+1$ points.

In the present paper, we study the superhedging problem under the framework of the guaranteed deterministic approach for a case of convex payoff functions and a market model where compact sets of possible price increments that describe the uncertainty of price movements are convex. Our paper extends the results published in Reference [27], where a specific (and economically interesting) case proved that a call option with a convex payoff at maturity has, under some mild assumptions, a convex pricing function. In the framework of the model with convexity assumptions, we also obtain some delicate properties for optimal mixed strategies of the "market" and clarify the behavior of their supports (the general case was considered in Reference [8]). We provide a two-dimensional (We consider a rainbow option whose payoff depends on two underlying risky assets.) example that brings to light some particularities of this behavior. 


\section{Convex Payoff Functions}

An important and widespread real-life case is the convexity of option payoff functions $g_{t}$. In this case, with an additional (and natural from the perspective of economic applications) assumption of convexity $K_{t}(\cdot)$, which we will admit everywhere further, the supports of the optimal mixed strategies of the "market" reveal a particular behavior. This is due to the following property of the Bellman-Isaacs functions $v_{t}^{*}$.

Proposition 1. Let the (deterministic) price dynamics be of Markov type, representable in the multiplicative form: the recurrent relation (R1) applies to the (discounted) price at time tof one unit of risky asset $i \in\{1, \ldots, n\}$

$$
X_{t}^{i}=M_{t}^{i} X_{t-1}^{i}, M_{t}=\left(M_{t}^{1}, \ldots, M_{t}^{n}\right) \in \check{C}_{t}, t=1, \ldots, N,
$$

where $\check{C}_{t}$ are convex compact sets with non-void interior such that $\check{C}_{t} \subseteq(0, \infty)^{n}$. Suppose that there are no trading constraints, i.e., $D_{t}(\cdot) \equiv \mathbb{R}^{n}$, and the NDAO condition (no arbitrage opportunities) is satisfied. If the payoff functions on the American option $\bar{x}_{t} \mapsto g_{t}\left(\bar{x}_{t}\right), t=1, \ldots, N$ are convex, then the solutions of Bellman-Isaacs equations $\bar{x}_{t} \mapsto v_{t}^{*}\left(\bar{x}_{t}\right), t=N, \ldots, 1$ are also convex.

Proof. Note first, that for the considered model the NDAO condition is tantamount to $e \in \check{C}_{t}$, where $e=(1, \ldots, 1) \in \mathbb{R}^{n}$. Denote by $\Lambda(m)$ the diagonal matrix with main diagonal entries equal to $m^{1}, \ldots, m^{n}$. Considering $X_{t}$ as the vector-column of $n$ prices of risky assets, we can rewrite (R1) as follows:

$$
X_{t}=\Lambda\left(M_{t}\right) X_{t-1}, M_{t} \in \check{C}_{t}, t=1, \ldots, N .
$$

Using the representation (R2) of price dynamics, let us prove the assertion by induction. The convexity is immediate for $s=N$. By induction, suppose that $v_{s}^{*}$ are convex for $s=N, \ldots, t \geq 1$. The convexity of $v_{t-1}^{*}$ follows from the formula (R3), which is a direct consequence of Theorem 2 in [6]:

$$
v_{t-1}^{*}\left(\bar{x}_{t-1}\right)=g_{t-1}\left(\bar{x}_{t-1}\right) \bigvee \sup _{P \in \mathcal{P}^{*}(\check{C}), \int m P(d m)=e}\left[\int v_{t}^{*}\left(\left(\bar{x}_{t-1}, \Lambda(m) x_{t-1}\right) P(d m)\right],\right.
$$

where $\mathcal{P}^{*}(E)$ stands for the class of all the probability measures, concentrated on finite subsets of a (non-void) set $E$. The convexity is conserved when taking integral (in fact, convex combinations) and supremum in (R3), whence the required result.

Lemma 1. If $X \subseteq \mathbb{R}^{n}$ is a nonvoid convex compact, function $f$ is continuous convex on $X, z \in X$, and then the functional $Q \mapsto \int f d Q$ reaches its maximum on the set (Here, $\mathcal{P}(X)$ denotes the class of all probability measures defined on a Borel $\sigma$-algebra of X.)

$$
E(z)=\left\{Q \in \mathcal{P}(X): \int y Q(d y)=z\right\}
$$

for some distribution $Q^{*}$ concentrated on at most $n+1$ extreme point of $X$.

Proof. According to Remark 3.2, Item 2) in Reference [7], there is a distribution $Q^{\prime}$ concentrated on $k \leq n+1$ points of $X$, which can be represented as $Q^{\prime}=\sum_{i=1}^{k} q_{i} \delta_{x^{i}}$, where $q \in S_{k-1}, x^{1}, \ldots, x^{k} \in X$ maximizes (due to continuity of $f$, the function $Q \mapsto \int f d Q$ is also continuous (for weak topology on $\mathcal{P}(X))$ ) function $Q \mapsto \int f d Q$ on the (weakly) compact set $E(z)$. Let us build a distribution $Q^{\prime \prime}$, related to $Q^{\prime}$ and concentrated on the finite number of points from $\operatorname{ext}(X)$, where ext $(\cdot)$ denotes a set of extreme points. By the Krein-Milman theorem (see, for example, Theorem 4.2 of Reference [28].) and the Caratheodory theorem (see, for example, Theorem 2.4 of Reference [28]) for each point $x^{i}, t=1, \ldots, k$ 
can be specified $x^{i j} \in \operatorname{ext}(X)$ and $p_{i j} \geq 0, j=1, \ldots, n+1$, such as $\sum_{j=1}^{n+1} p_{i j}=1, \sum_{j=1}^{n+1} p_{i j} x^{i j}=x^{i}$, $t=1, \ldots, k$. Therefore,

$$
\begin{aligned}
\int f d Q^{\prime} & =\sum_{i=1}^{k} q_{i} f\left(x^{i}\right)=\sum_{i=1}^{k} q_{i} f\left(\sum_{j=1}^{n+1} p_{i j} x^{i j}\right) \\
& \leq \sum_{i=1}^{k} q_{i} \sum_{j=1}^{n+1} p_{i j} f\left(x^{i j}\right)=\sum_{i=1}^{k} \sum_{j=1}^{n+1} q_{i j} f\left(x^{i j}\right)=\int f d Q^{\prime \prime},
\end{aligned}
$$

where $q_{i j}=q_{i} p_{i j} \geq 0$ and $\sum_{i=1}^{k} \sum_{j=1}^{n+1} q_{i j}=1$.

Thus, the distribution $Q^{\prime \prime}=\sum_{i=1}^{k} \sum_{j=1}^{n+1} q_{i j} \delta_{x^{i j}}$ maximizes the functional $Q \mapsto \int f d Q$ on $E(z)$, and $Q^{\prime \prime}$ is concentrated in the finite number of points of $\operatorname{ext}(X)$. However, the maximum $Q \mapsto \int f d Q$ on the set of distributions concentrated on $\operatorname{ext}(X)$ with a given mean $z$, i.e., $\left\{Q \in \mathcal{P}(\operatorname{ext}(X)), \int y Q(d y)=z\right\}$, where $z \in \operatorname{conv}(\operatorname{ext}(X))=X$, is reached on distributions concentrated on at most $n+1$ points of $\operatorname{ext}(X)$; see Reference [7], Remark 3.2, Item 2.

Remark 1. Assume that NDSAUP holds and the functions $v_{t}^{*}(\cdot), t=0, \ldots, N$ are convex everywhere (since the functions $v_{t}^{*}(\cdot)$ are convex everywhere on $\left(\mathbb{R}^{n}\right)^{t}$, they are continuous (see, for example, [29], Corollary 10.1.1) on $\left.\left(\mathbb{R}^{n}\right)^{t}\right)$.

(1) Therefore, the condition (USC) from [5], i.e., the upper semicontinuity of $v_{t}^{*}(\cdot), t=0, \ldots, N$, is fulfilled, so [5], Proposition 3.3 is applicable, and hence, there is an equilibrium with mixed extension $\mathcal{P}\left(K_{t}(\cdot)\right)$; moreover, $\mathcal{P}_{t}^{\text {opt }}(\cdot) \neq \varnothing$.

(2) If the maximizer in expression (2.6) from [8] is unique, i.e., $\mathcal{P}_{t}^{\text {opt }}(\cdot)=\left\{Q_{t}^{*}(\cdot)\right\}$ is a one-point set, then by Lemma 1, $\operatorname{supp}\left(Q_{t}^{*}(\cdot)\right) \subseteq \operatorname{ext}\left(K_{t}(\cdot)\right)$. Applying a two-stage optimization defined by the relations (11) and (12) in [7] and taking into account Item 2 of Remark 3.2 in [7], we conclude that the number of points in support $\left|\operatorname{supp}\left(Q_{t}^{*}(\cdot)\right)\right| \leq n+1$. Moreover, if the conditions of Theorem 2.1 from [8] are fulfilled, the mapping $x \mapsto Q_{t}^{*}(x)$ is (weakly) continuous and $x \mapsto \operatorname{supp}\left(Q_{t}^{*}(\cdot)\right)$ is a lower semicontinuous multivalued mapping.

(3) In the case that $K_{t}(\cdot)$ are convex polyhedra, i.e., can be represented as a convex hull of a finite number of points ( according to Theorem 19.1 in [29], the polyhedrality of a convex set is equivalent to its finite generation; in the case of compactness, such a set coincides with the convex hull of a finite number of points; see also [28], Definition 2.2), the set of extreme points $\operatorname{ext}\left(K_{t}(\cdot)\right)$ is finite and $m=\left|\operatorname{ext}\left(K_{t}(\cdot)\right)\right| \geq n+1$; so $n+1$ of these $m$ points constitute the optimal mixed strategy support.

Proposition 2. Assume that the functions $v_{t}^{*}(\cdot) t=0, \ldots, N$ are convex everywhere on $\left(\mathbb{R}^{n}\right)^{t}$. Let the compact-valued mappings $K_{t}(\cdot)$ be continuous, convex-valued mappings, $D_{t}(\cdot)$ be weakly continuous (i.e., lower semicontinuous and closed (see the terminology in $\S 14$ in [30])), and functions $g_{t}(\cdot)$ be convex, $t=1, \ldots, N$. Suppose that the robust condition of no sure arbitrage with unbounded profit RNDSAUP and one of two following conditions hold:

(1) set $K_{t}(\cdot)$ is strictly convex $t=1, \ldots, N$;

(2) $K_{t}(x)$ is a convex polyhedron with a constant (independent of $x$ ) number of vertices (the set of vertices of a compact convex polyhedron coincides with the set of its extreme points), $t=1, \ldots, N$.

Then, the multivalued mapping $x \mapsto \mathcal{P}_{t}^{\text {opt }}(x) \cap \mathcal{P}^{n}\left(\operatorname{ext}\left(K_{t}(x)\right)\right) \neq \varnothing$ is upper semicontinuous.

Proof. If condition (1) of this proposition is fulfilled, then by Corollary 9.2 in Reference [30] (from Theorems 9.3 and 8.1), the multi-valued mapping $x \mapsto \operatorname{ext}\left(K_{t}(\cdot)\right)$ is $h$-continuous (For compact-valued mappings, $h$-continuity and continuity are equivalent; see Theorem 2.68 from Reference [31].). In 
general, we can only guarantee that this mapping is $h$-lower semicontinuous (see, for example, a relevant construction in Example 9.2 in Reference [30].), but if condition (2) of this proposition is fulfilled, using Lemma 2 in Reference [32], we obtain $h$-continuity for $x \mapsto \operatorname{ext}\left(K_{t}(x)\right)$. According to Theorem 2.1 in Reference [8], the multi-valued mapping $x \mapsto \mathcal{P}^{o p t}(x)$ is upper semicontinuous. Furthermore, similar to the end of the proof of Theorem 2.1 in Reference [8], using Lemma 2.1 in Reference [8], we obtain the upper semicontinuity of the multi-valued mapping

$$
x \mapsto \mathcal{P}^{n}\left(\operatorname{ext}\left(K_{t}(x)\right),\right.
$$

and using Proposition 2.45 in Reference [31], which is applicable because $\mathcal{P}_{t}^{o p t}(x) \cap \mathcal{P}^{n}\left(\operatorname{ext}\left(K_{t}(x)\right)\right) \neq$ $\varnothing$, by Theorem 2 in Reference [7], we prove that the multi-valued mapping

$$
x \mapsto \mathcal{P}_{t}^{o p t}(x) \cap \mathcal{P}^{n}\left(\operatorname{ext}\left(K_{t}(x)\right)\right.
$$

is upper semicontinuous.

Remark 2. Condition (2) from Proposition 2 is fulfilled, in particular, for the market model considered by V.N. Kolokoltsov in his article in Reference [16]; his model can be classified as multiplicative and homogeneous over time according to the terminology used in Reference [1]. Multiplicative factors $M_{t}$ determine the price dynamics $X_{t}$ according to the representation:

$$
X_{t}^{i}=M_{t}^{i} X_{t-1}^{i}, M_{t}=\left(M_{t}^{1}, \ldots, M_{t}^{n}\right) \in C_{t}(\cdot), t=1, \ldots, N,
$$

where $C_{t}(\cdot)$ is a non-empty compact subset $\mathbb{R}^{n} ;$ Kolokoltsev considered $C_{t}(\cdot) \equiv \check{C}$, where $\check{C}$ is a fixed rectangle, that is, a set $\check{C}=\prod_{i=1}^{n}\left[\alpha_{i}, \beta_{i}\right]$.

Moreover, it is shown in Reference [4], Proposition 2 that in this case, the multi-valued mapping $K_{t}(\cdot)$ is not only h-continuous but also the Lipschitz function (with respect to the Pompeiu-Hausdorff distance).

Proposition 3. Assume that the functions $v_{t}^{*}(\cdot) t=0, \ldots, N$ are convex everywhere on $\left(\mathbb{R}^{n}\right)^{t}$. Let there be no trading constraints; the condition of no sure arbitrage NDSA is fulfilled. Suppose that the functions $g_{t}(\cdot)$ are convex and $K_{t}(\cdot)$ are convex polyhedra, $t=1, \ldots, N$.

(1) If the condition

$$
0 \notin \operatorname{ri}(\operatorname{conv}(A)) \text { for any } A \subseteq \operatorname{ext}\left(K_{t}(\cdot)\right) \text {, such as }|A| \leq n,
$$

is fulfilled, then we have the following:

- there is an optimal mixed strategy $Q_{t}^{*}(\cdot)$ with zero mean and $\operatorname{supp}\left(Q_{t}^{*}(\cdot)\right) \subseteq \operatorname{ext}\left(K_{t}(\cdot)\right)$ satisfying the condition of maximum cardinality of support, i.e., $\left|\operatorname{supp}\left(Q_{t}^{*}(\cdot)\right)\right| \equiv n+1$;

- compacts $K_{t}(\cdot)$ are full-dimensional, i.e., $\operatorname{dim} K_{t}(\cdot)=n$; and

- the robust condition of no arbitrage opportunities RNDAO is fulfilled.

(2) If, in addition, $\mathcal{P}_{t}^{\text {opt }}(\cdot)$ contains a single element, i.e., $\mathcal{P}_{t}^{\text {opt }}(\cdot)=\left\{Q_{t}^{*}(\cdot)\right\}$, the compact-valued mappings $K_{t}(\cdot)$ are continuous, $t=1, \ldots, N$, then multivalued mapping $x \mapsto \operatorname{supp}\left(Q_{t}^{*}(\cdot)\right)$ can be decomposed into $n$ non-coincident continuous everywhere branches, each of which is a vertex of one of the $K_{t}(\cdot)$ $n$-simplex (the $n$-simplex is a solid polyhedron in $\mathbb{R}^{n}$ with $n+1$ vertices (which are the extreme points of this polyhedron)). containing 0 . (There can be several such $n$-simplexes).

\section{Proof.}

(1) In the absence of trading constraints, NDSAUP and NDSA equivalent; therefore, $\rho_{t}^{\prime}(\cdot)>-\infty$; moreover, $\rho_{t}^{\prime}(\cdot) \geq 0$ (see Section 1 of this article). Since, by Item 1 ) of Remark 1 , the game equilibrium 
takes place, that is, $\rho_{t}(\cdot)=\rho_{t}^{\prime}(\cdot)$, and moreover, there are optimal mixed market strategies that maximize the expression (8). Using Lemma 1 , we find that there is an optimal mixed strategy $Q_{t}^{*}(\cdot)$ with zero mean and $\operatorname{supp}\left(Q_{t}^{*}(\cdot)\right) \subseteq \operatorname{ext}\left(K_{t}(\cdot)\right)$, where $\left|\operatorname{supp}\left(Q_{t}^{*}(\cdot)\right)\right| \leq n+1$. On the other hand, for an optimal mixed strategy with zero mean, that is, $\int y Q_{t}^{*}(d y)=0$, by Theorem 1.49 in Reference [9], we conclude that $0 \in \operatorname{ri}\left(\operatorname{conv}\left(\operatorname{supp}\left(Q_{t}^{*}(\cdot)\right)\right)\right.$; from condition (11), therefore, $\left|\operatorname{supp}\left(Q_{t}^{*}(\cdot)\right)\right| \geq n+1$ follows. Therefore, due to condition (11), we obtain that for this mixed strategy, $\left|\operatorname{supp}\left(Q_{t}^{*}(\cdot)\right)\right|=n+1$.

Denote $n^{\prime}=\operatorname{dim}\left(\operatorname{supp}\left(Q_{t}^{*}(\cdot)\right)\right.$; because $\operatorname{supp}\left(Q_{t}^{*}(\cdot)\right) \subseteq K_{t}(\cdot)$, we have $n^{\prime} \leq \operatorname{dim} K_{t}(\cdot) \leq n$. Let us suppose that $n^{\prime}<n$. According to the Caratheodory theorem, there are at most $n^{\prime}+1$ points $x_{1}, \ldots, x_{k}$ of $S(x)$, such as $0 \in \operatorname{conv}\left(\left\{x_{1}, \ldots, x_{k}\right\}\right)$, such that there are natural numbers $m \leq k$ and $q_{i_{1}}>0, \ldots, q_{i_{m}}>0, \sum_{j=1}^{m} q_{i_{j}}=1$, such as $\sum_{j=1}^{m} q_{i_{j}} x_{i_{j}}=0$, and hence, $0 \in \operatorname{ri}\left(\operatorname{conv}\left(\left\{x_{i_{1}}, \ldots, x_{i_{m}}\right\}\right)\right.$; this contradicts assumption (11). Therefore, $n^{\prime}=n$. However, it is then also the case that $\operatorname{dim} K_{t}(\cdot)=n$, that is, $K_{t}(\cdot)$ are full-dimensional.

Since $\operatorname{dim}\left(\operatorname{supp}\left(Q_{t}^{*}(\cdot)\right)=\operatorname{dim} K_{t}(\cdot)\right.$ and $\int y Q_{t}^{*}(d y)=0$, then by Proposition 1 in Reference [6], the condition of no arbitrage opportunities $N D A O$ is equivalent to $0 \in \operatorname{ri}\left(\operatorname{conv}\left(K_{t}(\cdot)\right)=\operatorname{ri}\left(K_{t}(\cdot)\right)\right.$. Finally, from the full-dimensionality of $K_{t}(\cdot)$, it follows that $\operatorname{ri}\left(K_{t}(\cdot)\right)=\operatorname{int}\left(K_{t}(\cdot)\right)$, which means that $0 \in \operatorname{int}\left(K_{t}(\cdot)\right)$, which in turn is equivalent to condition RNDAO by Proposition 5.1 in Reference [2].

(2) Using Proposition 3.3 in Reference [8], we obtain that for $Q_{t}^{*}(\cdot)$, which we found in Item 1) of the proof, the mapping $x \mapsto \operatorname{supp}\left(Q_{t}^{*}(\cdot)\right)$ is continuous, and thus, this multi-valued mapping can be decomposed into $n+1$ continuous everywhere non-coincident branches: $\operatorname{supp}\left(Q_{t}^{*}(\cdot)\right)=$ $\left\{y_{t}^{1}(\cdot), \ldots, y_{t}^{n+1}(\cdot)\right\} \subseteq \operatorname{ext}\left(K_{t}(\cdot)\right)$; note that and $0 \in \operatorname{int}\left(\operatorname{conv}\left(\left\{y_{t}^{1}(\cdot), \ldots, y_{t}^{n+1}(\cdot)\right\}\right)\right.$ and $\operatorname{conv}\left(\left\{y_{t}^{1}(\cdot), \ldots, y_{t}^{n+1}(\cdot)\right\}\right)$ is a simplex.

Under the assumptions of Proposition 1, we illustrate the behavior of the support of the optimal mixed strategy (Now, it will be more convenient to denote an optimal mixed strategy by $Q_{t, \text {, instead of }}^{*}$ $Q_{t}^{*}(\cdot)$.) $Q_{t}^{*}$, concentrated on the extreme points $K_{t}(\cdot)$, using the example of a two-dimensional market model with no trading constraints, as described in Remark 2 (i.e., for $n=2$ ).

Example 1. The market dynamics are described by the following relations:

$$
\begin{array}{ll}
X_{t}^{1}=M_{t}^{1} X_{t-1}^{1}, & M_{t}^{1} \in\left[\alpha^{1}, \beta^{1}\right] ; \\
X_{t}^{2}=M_{t}^{2} X_{t-1}^{2}, & M_{t}^{2} \in\left[\alpha^{2}, \beta^{2}\right] .
\end{array}
$$

In this case, $K_{t}(\cdot)$ is rectangle; for $i=1,2$ denote $a^{i}\left(x_{t-1}\right)=x_{t-1}\left(\alpha^{i}-1\right), b^{i}\left(x_{t-1}\right)=x_{t-1}\left(\beta^{i}-1\right)$; then,

$$
K_{t}\left(\bar{x}_{t-1}\right)=K_{t}\left(x_{0}, \ldots, x_{t-1}\right)=\left[a^{1}\left(x_{t-1}\right), b^{1}\left(x_{t-1}\right)\right] \times\left[a^{2}\left(x_{t-1}\right), b^{2}\left(x_{t-1}\right)\right] .
$$

Assume that condition RNDAO is fulfilled, that is, $0 \in \operatorname{int}\left(K_{t}(\cdot)\right)$, which is tantamount to $\alpha^{1}<1<\beta^{1}$ and $\alpha^{2}<1<\beta^{2}$.

For the support $S_{t}(\cdot)=\operatorname{supp}\left(Q_{t,}^{*}\right)$ of an optimal mixed strategy, concentrated on extreme points, that is, the vertices of a rectangle $K_{t}(\cdot)$, condition $R N D A O$ eliminates the case in which $\left|S_{t}(\cdot)\right|=1$; moreover, for the case in which $\left|S_{t}(\cdot)\right|=2$, it also excludes the possibility that the support $S_{t}(\cdot)$ consists of the end points of any side of the rectangle; so if the support $S_{t}(\cdot)$ is concentrated on two points, then these points are the end points of the diagonal (closed segments connecting opposite vertices of the rectangle $\left.K_{t}(\cdot)\right)$. We refer to the diagonal connecting points $\left(a^{1}\left(x_{t-1}\right), a^{2}\left(x_{t-1}\right)\right)$ and $\left(b^{1}\left(x_{t-1}\right), b^{2}\left(x_{t-1}\right)\right)$ and the diagonal connecting $\left(a^{1}\left(x_{t-1}\right), b^{2}\left(x_{t-1}\right)\right)$ and $\left(b^{1}\left(x_{t-1}\right), a^{2}\left(x_{t-1}\right)\right)$ as the positive and negative (This terminology is chosen because for a distribution with a support consisting of two or three extreme points of $K_{t}(\cdot)$ containing this diagonal, random variables that are components of a random vector with this distribution have a positive or negative 
correlation coefficient, respectively.) connecting points, respectively. Furthermore, it is easy to check that the point $\mathbf{0}=(0,0)$ lies on the positive diagonal if and only if

$$
\frac{\beta^{1}-1}{\beta^{1}-\alpha^{1}}=\frac{\beta^{2}-1}{\beta^{2}-\alpha^{2}} \in(0,1)
$$

denote this number by $p$. Point $\mathbf{0}=(0,0)$ lies on the negative diagonal if and only if

$$
\frac{\beta^{1}-1}{\beta^{1}-\alpha^{1}}=\frac{1-\alpha^{2}}{\beta^{2}-\alpha^{2}} \in(0,1)
$$

denote this number by $q$. If point $\mathbf{0}=(0,0)$ lies at the intersection of the diagonals, that is, (12) and (13) are fulfilled simultaneously, then $p=q=1 / 2$. Four more possible cases arise when $\left|S_{t}(\cdot)\right|=3$, that is, when both (12) and (13) are not fulfilled:

$$
\frac{\beta^{2}-1}{\beta^{2}-\alpha^{2}} \neq \frac{\beta^{1}-1}{\beta^{1}-\alpha^{1}} \neq \frac{1-\alpha^{2}}{\beta^{2}-\alpha^{2}} .
$$

For this example, this is exactly condition (11) (this takes as given that the point $\mathbf{0}=(0,0)$ is not a vertex of the rectangle $K_{t}(\cdot)$ and does not belong to its sides) from Proposition 3. Note that the coefficients $\alpha^{1}, \beta^{1}, \alpha^{2}$ and $\beta^{2}$ are market model parameters and are not related to payoff function values. Summarizing the various cases, we find that the optimal mixed strategy, concentrated on at most three extreme points (vertices) of $K_{t}(\cdot)$, will be concentrated:

(1) on two points when (12) and (13) are fulfilled simultaneously, that is, $\mathbf{0}=(0,0)$ lies at the intersection of the diagonals; two points always lie on one of the diagonals;

(2) on two or three points if either (12) or (13), that is, point $\mathbf{0}=(0,0)$ lies on one of the diagonals; two points always lie on one of the diagonals; and

(3) on three points if both (12) and (13) are not fulfilled, that is, when (14) holds, the condition (11) is fulfilled in this case.

To determine the optimal mixed strategy with $S_{t}(\cdot) \subseteq \operatorname{ext}\left(K_{t}(\cdot)\right)$ and $\left|S_{t}(\cdot)\right| \leq 3$, we need information about the values of the Bellman function $y \mapsto w_{t}(\cdot, y)$ at the vertices of the rectangle $K_{t}(\cdot)$. According to the results in Reference [7], Sections 4 and 5, we construct the concave hull of the function $y \mapsto w_{t}(\cdot, y)$. To do so, it suffices to determine the "dominant" diagonal by checking the values that affine functions defined on the diagonals by the values of the Bellman function $y \mapsto w_{t}(\cdot, y)$ at the end points of the corresponding diagonal take at the diagonals' intersection point, that is, comparing

$$
\frac{1}{2}\left[w_{t}\left(\cdot,\left(a^{1}\left(\bar{x}_{t-1}\right), a^{2}\left(\bar{x}_{t-1}\right)\right)\right)+w_{t}\left(\cdot,\left(b^{1}\left(\bar{x}_{t-1}\right), b^{2}\left(\bar{x}_{t-1}\right)\right)\right)\right]
$$

(the value for the positive diagonal) and

$$
\frac{1}{2}\left[w_{t}\left(\cdot,\left(a^{1}\left(\bar{x}_{t-1}\right), b^{2}\left(\bar{x}_{t-1}\right)\right)\right)+w_{t}\left(\cdot,\left(b^{1}\left(\bar{x}_{t-1}\right), a^{2}\left(\bar{x}_{t-1}\right)\right)\right)\right]
$$

(the value for the negative diagonal). When one of the expressions (15) and (16) takes a greater value, it corresponds to the dominant diagonal; when expressions (15) and (16) take the same value, the concave hull of the Bellman function is an affine function.

According to the results of Reference [33], a solid polyhedron $M_{k}$ in $\mathbb{R}^{n}$ with $k$ vertices $(k \geq n+1)$ can be represented as a simplicial decomposition union of (this is the minimum possible number of n-simplexes in simplicial decomposition.) $k-n$ subsets and $n$-simplexes ( $n$-simplex is a solid polyhedron in $\mathbb{R}^{n}$ with $n+1$ vertexes.) with vertices that are vertices of the polyhedron, so that the interiors of these n-simplixes would be non-intersecting. For our example, there are two such decompositions: the division by the positive diagonal into two 3-simplexes (i.e., a triangle) or by the negative diagonal into two other 3-simplexes; accordingly, we call these decompositions positive and negative. 
If one of the expressions (15) and (16) is strictly greater than the other, then the decomposition corresponding to the dominant diagonal is of interest, and the concave hull is a piecewise affine function on triangles having the dominant diagonal as their common side. Recall that in accordance with the results of Reference [7] the value $\rho_{t-1}^{\prime}(\cdot)$ is defined as the value of the concave hull function $y \mapsto w_{t}(\cdot, y)$ at point $y=0$. By Theorem 1 in Reference [6], there is an equilibrium in the class of mixed strategies concentrated on a finite number of points. Note that since RNDAO implies NDSA, then according to the results in Reference [5] (see the reasoning after Remark 7), the European option can be considered a special case of the American option when $g_{t}(\cdot) \equiv 0$ for $t=1, \ldots, N-1$ and $g_{N}=g$, where $g$ is a terminal payoff function. Therefore, in the case of inequality

$$
\begin{gathered}
w_{t}\left(\cdot,\left(a^{1}\left(x_{t-1}\right), a^{2}\left(x_{t-1}\right)\right)\right)+w_{t}\left(\cdot,\left(b^{1}\left(x_{t-1}\right), b^{2}\left(x_{t-1}\right)\right)\right)> \\
w_{t}\left(\cdot,\left(a^{1}\left(x_{t-1}\right), b^{2}\left(x_{t-1}\right)\right)\right)+w_{t}\left(\cdot,\left(b^{1}\left(x_{t-1}\right), a^{2}\left(x_{t-1}\right)\right)\right)
\end{gathered}
$$

The positive diagonal dominates. If (12) is fulfilled, that is, $\mathbf{0}=(0,0)$ is on the positive diagonal, then

$$
Q_{t, \bar{x}_{t-1}}^{*}=p \delta_{\left(a^{1}\left(x_{t-1}\right), a^{2}\left(x_{t-1}\right)\right)}+(1-p) \delta_{\left(b^{1}\left(x_{t-1}\right), b^{2}\left(x_{t-1}\right)\right)} .
$$

If

$$
\frac{\beta^{1}-1}{\beta^{1}-\alpha^{1}}>\frac{\beta^{2}-1}{\beta^{2}-\alpha^{2}}
$$

and, therefore, the point $\mathbf{0}=(0,0)$ is in the interior of the "upper triangle", that is, the triangle that has vertex $\left(a^{1}\left(x_{t-1}\right), b^{2}\left(x_{t-1}\right)\right)$ and positive diagonal as its side, then

$$
Q_{t, \bar{x}_{t-1}}^{*}=p_{1} \delta_{\left(a^{1}\left(x_{t-1}\right), a^{2}\left(x_{t-1}\right)\right)}+p_{2} \delta_{\left(b^{1}\left(x_{t-1}\right), b^{2}\left(x_{t-1}\right)\right)}+p_{3} \delta_{\left(a^{1}\left(x_{t-1}\right), b^{2}\left(x_{t-1}\right)\right)},
$$

where

$$
\begin{gathered}
p_{1}=\frac{1-\alpha^{1}}{\beta^{1}-\alpha^{1}}>0, \\
p_{2}=\frac{\beta^{2}-1}{\beta^{2}-\alpha^{2}}>0, \\
p_{3}=1-p_{1}-p_{2}>0 .
\end{gathered}
$$

If the point $\mathbf{0}=(0,0)$ is in the interior of the opposite ("lower") triangle in the rectangle decomposition, which is the case if

$$
\frac{\beta^{1}-1}{\beta^{1}-\alpha^{1}}<\frac{\beta^{2}-1}{\beta^{2}-\alpha^{2}}
$$

then

$$
Q_{t, \bar{x}_{t-1}}^{*}=p_{1}^{\prime} \delta_{\left(a^{1}\left(x_{t-1}\right), a^{2}\left(x_{t-1}\right)\right)}+p_{2}^{\prime} \delta_{\left(b^{1}\left(x_{t-1}\right), b^{2}\left(x_{t-1}\right)\right)}+p_{3}^{\prime} \delta_{\left(b^{1}\left(x_{t-1}\right), a^{2}\left(x_{t-1}\right)\right)^{\prime}}
$$

where

$$
\begin{gathered}
p_{1}^{\prime}=\frac{\beta^{1}-1}{\beta^{1}-\alpha^{1}}>0, \\
p_{2}^{\prime}=\frac{1-\alpha^{2}}{\beta^{2}-\alpha^{2}}>0, \\
p_{3}^{\prime}=1-p_{1}^{\prime}-p_{2}^{\prime}>0 .
\end{gathered}
$$

If we have the case of the opposite inequality,

$$
\begin{gathered}
w_{t}\left(\cdot,\left(a^{1}\left(x_{t-1}\right), a^{2}\left(x_{t-1}\right)\right)\right)+w_{t}\left(\cdot,\left(b^{1}\left(x_{t-1}\right), b^{2}\left(x_{t-1}\right)\right)<\right. \\
w_{t}\left(\cdot,\left(a^{1}\left(x_{t-1}\right), b^{2}\left(x_{t-1}\right)\right)\right)+w_{t}\left(\cdot,\left(b^{1}\left(x_{t-1}\right), a^{2}\left(x_{t+1}\right)\right)\right),
\end{gathered}
$$


then the negative diagonal dominates. Similar to the previous case, if (13) is fulfilled, that is, point $\mathbf{0}=(0,0)$ is on the negative diagonal, then

$$
Q_{t, \bar{x}_{t-1}}^{*}=q \delta_{\left(a^{1}\left(x_{t-1}\right), b^{2}\left(x_{t-1}\right)\right)}+(1-q) \delta_{\left(b^{1}\left(x_{t-1}\right), a^{2}\left(x_{t-1}\right)\right)} .
$$

If

$$
\frac{\beta^{1}-1}{\beta^{1}-\alpha^{1}}>\frac{1-\alpha^{2}}{\beta^{2}-\alpha^{2}}
$$

then, point $\mathbf{0}=(0,0)$ lies inside a triangle with vertices $\left(a^{1}\left(x_{t-1}\right), b^{2}\left(x_{t-1}\right)\right),\left(b^{1}\left(x_{t-1}\right), a^{2}\left(x_{t-1}\right)\right.$ and $\left(a^{1}\left(x_{t-1}\right), a^{2}\left(x_{t-1}\right)\right)$, that is, it is in the "lower" triangle, and then,

$$
Q_{t, \bar{x}_{t-1}}^{*}=q_{1} \delta_{\left(a^{1}\left(x_{t-1}\right), b^{2}\left(x_{t-1}\right)\right)}+q_{2} \delta_{\left(b^{1}\left(x_{t-1}\right), a^{2}\left(x_{t-1}\right)\right)}+q_{3} \delta_{\left(a^{1}\left(x_{t-1}\right), a^{2}\left(x_{t-1}\right)\right)^{\prime}}
$$

where

$$
\begin{array}{r}
q_{1}=\frac{1-\alpha^{2}}{\beta^{2}-\alpha^{2}}>0, \\
q_{2}=\frac{1-\alpha^{1}}{\beta^{1}-\alpha^{1}}>0, \\
q_{3}=1-q_{1}-q_{2}>0 .
\end{array}
$$

If

$$
\frac{\beta^{1}-1}{\beta^{1}-\alpha^{1}}<\frac{1-\alpha^{2}}{\beta^{2}-\alpha^{2}}
$$

then point $\mathbf{0}=(0,0)$ lies in the "upper" (from the negative diagonal) triangle, the vertices of which are points $\left(a^{1}\left(x_{t-1}\right), b^{2}\left(x_{t-1}\right)\right) ;\left(b^{1}\left(x_{t-1}\right), a^{2}\left(x_{t-1}\right)\right.$ and $\left(b^{1}\left(x_{t-1}\right), b^{2}\left(x_{t-1}\right)\right)$, and then,

$$
Q_{t, \bar{x}_{t-1}}^{*}=q_{1}^{\prime} \delta_{\left(a^{1}\left(x_{t-1}\right), b^{2}\left(x_{t-1}\right)\right)}+q_{2}^{\prime} \delta_{\left(b^{1}\left(x_{t-1}\right), a^{2}\left(x_{t-1}\right)\right)}+q_{3}^{\prime} \delta_{\left(b^{1}\left(x_{t-1}\right), b^{2}\left(x_{t-1}\right)\right)}
$$

where

$$
\begin{array}{r}
q_{1}^{\prime}=\frac{\beta^{1}-1}{\beta^{1}-\alpha^{1}}>0, \\
q_{2}^{\prime}=\frac{\beta^{2}-1}{\beta^{2}-\alpha^{2}}>0, \\
q_{3}^{\prime}=1-q_{1}^{\prime}-q_{2}^{\prime}>0 .
\end{array}
$$

Finally, in the case of equality

$$
\begin{gathered}
w_{t}\left(\cdot,\left(a^{1}\left(x_{t-1}\right), a^{2}\left(x_{t-1}\right)\right)\right)+w_{t}\left(\cdot,\left(b^{1}\left(x_{t-1}\right), b^{2}\left(x_{t-1}\right)\right)=\right. \\
w_{t}\left(\cdot,\left(a^{1}\left(x_{t-1}\right), b^{2}\left(x_{t-1}\right)\right)\right)+w_{t}\left(\cdot,\left(b^{1}\left(x_{t-1}\right), a^{2}\left(x_{t-1}\right)\right)\right),
\end{gathered}
$$

because the concave hull of the Bellman function is affine, the optimal mixed strategy with the support $S_{t}(\cdot) \subseteq$ $\operatorname{ext}\left(K_{t}(\cdot)\right)$ is not unique: any probability measure with the support described above in cases (1), (2) and (3) and zero mean will be optimal.

Thus, what is decisive in this case is the position of the point $\mathbf{0}=(0,0)$. If (11) is fulfilled, that is, (14), then $\mathbf{0}$ lies in the interior of the intersection of the two triangles, one from the positive decomposition of the rectangle and the other from the negative decomposition. (In this case, there are two possible variants for the support, which are concentrated in three vertices of the triangle.) If either (12) or (13) is fulfilled, then the optimal distributions are concentrated at two end points of the diagonal, on which the point $\mathbf{0}=(0,0)$ lies, or in the vertices of one of the triangles, one side of which is the opposite diagonal. If both (12) and (13) are fulfilled 
simultaneously, that is, point $\mathbf{0}=(0,0)$ lies at the intersection of the diagonals, the distribution is concentrated at the end points of a diagonal, and assigning to its end points the probability $1 / 2$ is optimal.

From an algebraic perspective, conditions (17)-(19) can be interpreted as conditions (The definitions can be found, for example, in Reference [34]; see Definition 2.3.1.) for the strict supermodularity, strict submodularity and modularity of the function $y \mapsto w_{t}(\cdot, y)$ on the lattice (See, for example, Reference ([35] Chapter 1, Sections 1 and 4)) of the four extreme points $K_{t}(\cdot)$ with coordinate-wise comparison (i.e., for $y=\left(y^{1}, y^{2}\right)$ and $z=\left(z^{1}, z^{2}\right)$, the relation $y \leq z$ means that $y^{1} \leq y^{2}$ and $z^{1} \leq z^{2}$ ).

As an alternative to the geometric approach based on the construction of a concave hull, one can use simple probabilistic reasoning. Denote by $\mathfrak{M}_{t}(\cdot)$ the class of all distributions with zero mean, such that the distribution's support is concentrated at two or three extreme points of the rectangle $K_{t}(\cdot)$. If $Q \in \mathfrak{M}_{t}(\cdot)$ and $\operatorname{supp}(Q)$ contain the ends of the nondominant diagonal, then $\mathfrak{M}_{t}(\cdot)$ and there is a distribution $Q^{\prime}$ with a greater value than the Bellman function. Indeed, let $\pi>0$ be the minimum probability of two points being on a nondominant diagonal. Denote the corresponding end point of the diagonal as $y_{1}$, another point of this diagonal as $y_{2}$, and the remaining point $\operatorname{supp}(Q)$, lying on the dominant diagonal, as $y_{3}$. Represent $Q$ in the form of (If $\pi=1 / 2$, then as $Q_{1}$, we can take an arbitrary distribution with $\operatorname{supp}\left(Q_{1}\right) \subseteq\left\{y_{2}, y_{3}\right\}$; in the case of $\pi<1 / 2$, the distribution $Q_{1}$ is uniquely determined.)

$$
Q=\pi \delta_{y_{1}}+\pi \delta_{y_{2}}+(1-2 \pi) Q_{1}
$$

and $\operatorname{supp}\left(Q_{1}\right) \subseteq\left\{y_{2}, y_{3}\right\}$ thanks to the choice $\pi$. Then, if $y_{4}$-one more point of the dominant diagonal-then the distribution

$$
Q^{\prime}=\pi \delta_{y_{3}}+\pi \delta_{y_{4}}+(1-2 \pi) Q_{1}
$$

has zero mean, and its support $\operatorname{supp}\left(Q^{\prime}\right) \subseteq\left\{y_{2}, y_{3}, y_{4}\right\}$. Obviously,

$$
\int w_{t}(\cdot, y) Q(d y)-\int w_{t}(\cdot, y) Q^{\prime}(d y)=\pi\left[\left(w_{t}\left(\cdot, y_{1}\right)+w_{t}\left(\cdot, y_{2}\right)\right)-\left(w_{t}\left(\cdot, y_{3}\right)+w_{t}\left(\cdot, y_{4}\right)\right)\right]<0
$$

\section{Remark 3.}

(1) A kind of "degeneration" observed in the above example, that is the case in which $\left|S_{t}(\cdot)\right|=2$, can be regarded as an exceptional case, as small "perturbations" of one of the parameters $\alpha^{1}, \beta^{1}, \alpha^{2}$ or $\beta^{2}$ leads to optimal mixed strategy with $\left|S_{t}(\cdot)\right|=3$.

(2) The effect observed in Example 1 in the case in which the point $\mathbf{0}$ lies at the intersection of the diagonals is a characteristic of the dimension $n \geq 2$. In a certain way, this is related to Steinitz's theorem [36]: if $A \subseteq \mathbb{R}^{n}$ and $x \in \operatorname{int}(\operatorname{conv}(A))$, then there is a $2 n$ point $A$, ithat is, $\left\{x_{1}, \ldots, x_{2 n}\right\} \subseteq A$, such as $x \in$ $\operatorname{int}\left(\operatorname{conv}\left(\left\{x_{1}, \ldots, x_{2 n}\right\}\right)\right.$. In the case of $n=1$, we have $n+1=2 n$, and at $n \geq 2$, we have $n+1<2 n$. In Example 1 (for $n=2$ ), one cannot guarantee that the point 0 lying in the interior of the convex hull of four points will lie in the interior of the hull of some three of the four points.

(3) In Reference [14], the problem of guaranteed super-hedging for a multiplicative Markov-type market model is considered (see the terminology in Reference [1]) under the assumption of no trading constraints. For the European rainbow option, the payoff function $g(\cdot)$ in Reference [14] depends only on the prices of risky assets at a terminal point in time $N$, that is, for some function $\psi$, there is the representation

$$
g\left(\bar{x}_{N}\right)=\psi\left(x_{N}\right)
$$

Note that for such an option, as well as for the American option, when the payoff functions $g_{t}(\cdot)$ are representable as

$$
g_{t}\left(\bar{x}_{t}\right)=\varphi_{t}\left(x_{t}\right), t=1, \ldots, N,
$$

for some functions $\varphi_{t}$, the random process corresponding to conditional distributions $P\left(X_{t} \in A \mid \bar{X}_{t}=\bar{x}_{t}\right)=$ $Q_{t, \bar{x}_{t}}^{*}$ will be Markovian. In Reference [14], explicit formulas are obtained for solutions when (20) is satisfied, in a two-dimensional case $(n=2)$, under the assumptions (in addition, in Reference [14], the monotony of the payoff function is imposed) of convexity of the payoff function $g$ and its submodularity. In Reference [37], as for 
case (20), explicit solutions are obtained for a similar problem in the three-dimensional case $(n=3)$, under the assumption of the convexity and submodularity of the payoff function. In Chapter 13 in Reference [16] (written by V.N. Kolokoltsov), the main Theorem 13.2 is demonstrated for the n-dimensional case when fulfilled (20). Note that the Bellman function preserves the submodularity property for a number of options (the expressions for the payoff functions of these (and other) options are listed in the table at the end of the article), for example, "best of asset or cash", "call on max", "multistrike", for which (20) also holds, that allow the selection of the options from the mixed strategies regarded as "candidates for optimality".

Under the assumptions of Proposition 1, a particularly simple situation arises in the one-dimensional case, that is, when $n=1$. In this case, $K_{t}(\cdot)$ has the form $\left[a_{t}(\cdot), b_{t}(\cdot)\right]$, where $a_{t}(\cdot) \leq b_{t}(\cdot)$. The concave hull convex of a function $f$ on a closed interval $[a, b]$ is the affine function that coincides with $f$ in the extreme points of this interval:

$$
f^{*}(x)=f(a)+\frac{f(b)-f(a)}{b-a}(x-a) .
$$

Thus, the maximum of the functional $Q \mapsto \int f d Q$ subject to $\int y Q(d y)=z \in(a, b)$ is attained for a distribution concentrated on the set $\{a, b\}$ of extreme points of the interval $[a, b]$. This fact was first reported, to the best of our knowledge, in the first edition of the book by Dana and Jeanblanc-Picqué 1994 (second edition [38]).

Taking this fact and Proposition 1 into account, one can easily write the Bellman equations (see Reference ([7] Formula (6.2)))

$$
\begin{gathered}
v_{N}^{*}(\cdot)=g_{N}(\cdot), \\
v_{t-1}^{*}(\cdot)=g_{t-1}(\cdot) \vee \int w_{t}(\cdot, y) Q_{t, \cdot}^{*}(d y), t=N, N-1, \ldots, 1,
\end{gathered}
$$

and find the relevant hedging strategies.

Example 2. Consider the multiplicative model from Example 3.2 in Reference [2] (in the one-dimensional case with no trading constraints) when assuming the condition of non-arbitrage NDAO. Assume that the payoff functions $g_{t}, t=1, \ldots, N$ are convex. Then, the most unfavorable scenario (mixed market strategy) will be the case when the conditional distributions of price increments $\Delta X_{t}$ are concentrated on points $x_{t}(\alpha-1)$ and $x_{t}(\beta-1)$ with probabilities $q_{-}$and $q_{+}$determined by a system of linear equations, which in this case has the form:

$$
\begin{gathered}
q_{-} x_{t}(\alpha-1)+q_{+} x_{t}(\beta-1)=0 \\
q_{-}+q_{+}=1
\end{gathered}
$$

and thus, $q_{-}=\frac{(1-\alpha)}{(\beta-\alpha)}, q_{+}=\frac{(\beta-1)}{(\beta-\alpha)}$. The NDAO condition in this case is equivalent to $\alpha<1<\beta$; hence, $q_{-}$and $q_{+}$lie in the interval $(0,1)$, which corresponds to their probabilistic interpretation. Note that in this case, the joint distribution of the random variables $M_{t}=\frac{X_{t}}{X_{t-1}}, t=1, \ldots, N$ is such that they are independent (In other words, there is independence of logarithmic increments.). This is because restrictions on $M_{t}$ have the form $M_{t} \in[\alpha, \beta]$, independent of the price history, that is, the price dynamics refer to a multiplicative-independent type. 
The Bellman equations (23) have the following form:

$$
\begin{aligned}
v_{N}^{*}\left(x_{0}, \ldots, x_{N}\right) & =g_{N}\left(x_{0}, \ldots, x_{N}\right), \\
v_{t-1}^{*}\left(x_{0}, \ldots, x_{t-1}\right) & =g_{t-1}\left(x_{0}, \ldots, x_{t-1}\right) \\
& \vee\left[q_{-} v_{t}^{*}\left(x_{0}, \ldots, x_{t-1}, x_{t-1} \alpha\right)\right. \\
& +q_{+} v_{t}^{*}\left(x_{0}, \ldots, x_{t-1}, x_{t-1} \beta\right),
\end{aligned}
$$

where $t=1, \ldots, N$. In this case, taking into account (22), the optimal hedging strategy $h_{t}(\cdot)$ at time t will be

$$
\begin{array}{r}
h_{t}^{*}\left(x_{0}, \ldots, x_{t-1}\right)=\frac{1}{x_{t-1}(\beta-\alpha)}\left[g_{t-1}\left(x_{0}, \ldots, x_{t-1}\right) \vee v_{t}^{*}\left(x_{0}, \ldots, x_{t-1}, x_{t-1} \beta\right)\right. \\
\left.-g_{t-1}\left(x_{0}, \ldots, x_{t-1}\right) \vee v_{t}^{*}\left(x_{0}, \ldots, x_{t-1}, x_{t-1} \alpha\right)\right] .
\end{array}
$$

In the case of European options, when $g_{1}=\ldots=g_{N-1}=0, g_{N}=g$ is a convex function, we obtain difference equations:

$$
\begin{gathered}
v_{N}^{*}(\cdot)=g_{N}(\cdot), \\
v_{t-1}^{*}(\cdot)=q_{-} v_{t}^{*}\left(\cdot, x_{t-1} \alpha\right)+q_{+} v_{t}^{*}\left(\cdot, x_{t-1} \beta\right), t=1, \ldots, N,
\end{gathered}
$$

and (25) is simplified to formula

$$
h_{t}^{*}(\cdot)=\frac{1}{x_{t-1}(\beta-\alpha)}\left[v_{t}^{*}\left(\cdot, x_{t-1} \beta\right)-v_{t}^{*}\left(\cdot, x_{t-1} \alpha\right)\right] \text {. }
$$

In particular, for European options with the payoff function (at the terminal moment $N$ ) of the form $g\left(\bar{x}_{N}\right)=\left(x_{N}-B\left(\bar{x}_{N}\right)\right)_{+}$, that is, a call option with the exercise price $B\left(\bar{x}_{t}\right)$ dependent on price trajectory $\bar{x}_{t}$ or $g\left(\bar{x}_{N}\right)=\left(S\left(\bar{x}_{N}\right)-x_{N}\right)_{+}$, that is, a put option with the exercise price $S\left(\bar{x}_{t}\right)$ dependent on price trajectory $\bar{x}_{t}$, the convexity of $g$ is valid, for example, for Asian options, lookback options and plain vanilla options. In the case of Asian options $B\left(\bar{x}_{N}\right)=S\left(\bar{x}_{N}\right)=\frac{1}{N+1} \sum_{t=0}^{N} x_{t}$, for lookback options, $B\left(\bar{x}_{N}\right)=\bigwedge_{t=0}^{N} x_{t}$ and $S\left(\bar{x}_{N}\right)=\bigvee_{t=0}^{N} x_{t}$, and for plain vanilla options, $B\left(\bar{x}_{N}\right)$ and $S\left(\bar{x}_{N}\right)$ are constant.

Note that for Asian options and lookback options, if we denote $A_{t}=\frac{1}{t+1} \sum_{s=0}^{t} x_{s}, L_{t}=\bigwedge_{s=0}^{t} x_{s}$ and $N_{t}=\bigvee_{s=0}^{t} x_{s}$, then the processes $\left(X_{t}, A_{t}\right),\left(X_{t}, L_{t}\right)$ and $\left(X_{t}, N_{t}\right)$ are Markovian and in difference Equations (26) for the Asian option and the lookback option, it suffices to leave only two variables instead of the entire price trajectory. For example, for a lookback call option, the difference Equations (26) can be represented as

$$
v_{t}^{*}\left(x_{0}, \ldots, x_{t}\right)=v_{t}\left(x_{t}, \bigwedge_{s=0}^{t} x_{s}\right)
$$

where $v_{t}$ satisfy the recurrence equations for $t=N, N-1, \ldots$ :

$$
\begin{gathered}
v_{N}\left(x_{N}, l_{N}\right)=\left(x_{N}-l_{N}\right) \\
v_{t-1}\left(x_{t-1}, l_{t-1}\right)=q_{-} v_{t}\left(x_{t-1} \alpha, l_{t} \wedge x_{t-1} \alpha\right)+q_{+} v_{t}\left(x_{t-1} \beta, l_{t} \wedge x_{t-1} \beta\right), \\
t=1, \ldots, N .
\end{gathered}
$$

Note also that the price movement model, which corresponds to independent $M_{t}, t=1, \ldots, N$, accepting values $\alpha$ and $\beta$ with probabilities $q_{-}$and $q_{+}$, will coincide with the Cox-Ross-Rubinstein model (This is also called the binomial model; see Reference [39]) if $x_{0}$ is fixed and the numbers $\alpha$ and $\beta$ are related by the ratio $\alpha \beta=1$; the advantage of the Cox-Ross-Rubinstein model is that lattice recombination occurs on a logarithmic 
scale, that is, the random variables $Z_{t}=\ln \left(\frac{X_{t}}{X_{0}}\right)=\sum_{s=1}^{t} \ln M_{s}, t=1, \ldots, N$, take values in the lattice (the lattice forms a discrete additive subgroup of the group $\mathbb{R}$.) $\{k d, k=0, \pm 1, \pm 2, \ldots\}$, where $d=\ln \beta=-\ln \alpha$. This reduces the number of possible values of $Z_{t}$ from $2^{t}$ to $t+1$. Moreover, the probability that $Z_{t}$ takes value $k d$ equals (Here, $C_{n}^{k}$ is the binomial coefficient, the number of combinations of $n$ and $k$.) $C_{t}^{(t+k) / 2} q_{+}^{(t+k) / 2} q_{-}^{(t-k) / 2}$. Note that in our case, recombination can be achieved by requiring the numbers $\ln \alpha$ and $\ln \beta$ to be rationally commensurable, that is, their ratio $\frac{\ln \alpha}{\ln \beta}$ would be a rational number $r=-\frac{n}{m}$, where $n$ and $m$ are mutually prime natural numbers (i.e., the ratio $\frac{n}{m}$ is irreducible). Determining the lattice step as $d=\frac{\ln \beta}{m}=-\frac{\ln \alpha}{n}$, we obtain that all values of a random variable $Z_{t}, t=0,1, \ldots$ (without time limit) lie on the lattice $\{k d, k=0, \pm 1, \pm 2, \ldots\}$, and there is no smaller sublattice on which all possible values of $Z_{t}$ would lie (because $n$ and $m$ are mutually simple. (See, for example, Reference [40].). Since any real number can be approximated by rational numbers of the form $\frac{n}{m}$ with accuracy not exceeding $\frac{1}{m}$, the initial problem with given $\alpha$ and $\beta$ can be replaced by and approximation with $\alpha^{\prime}$ and $\beta^{\prime}$, where $\alpha^{\prime}$ and $\beta^{\prime}$ will be rationally commensurable. From the perspective of numerical algorithms, recombination makes sense for lookback options; however, it is meaningless for Asian options because the exercise price remains on the lattice in the first case but not in the second case.

Remark 4. In the one-dimensional case, the above property (22) for the convex payoff function, under the no trading constraints assumption and if the condition of no arbitrage opportunities NDAO holds, has the following economic interpretation for a one-step problem. Buying an option with a convex payoff function can be interpreted as "buying volatility" because it protects from the most "unfavorable" scenario, which is the most "volatile" price movement when price increments take values at the extreme points of the segments of price limits (in any case, if volatility is measured with the help of the standard deviation of the price increment, then among all the risk-neutral probabilities, its maximum is attained in this particular case). Buying an option with a concave payoff function can be interpreted as "selling volatility", as protection against the most "unfavorable" scenario, which is the lack of price volatility; for the corresponding risk-neutral probability, price increments are zero (with zero volatility measured as the standard deviation of price increments).

It is possible to present many examples of rainbow options, that is, when the number of risky assets is greater than one, (The number of risky assets is also called the number of colors (of the rainbow).) with convex payoff functions. These options, for example, include European rainbow options in Table 1.

Table 1. Examples of European rainbow options with convex payoff functions.

\begin{tabular}{ll}
\hline Option Type & Payoff Function \\
\hline "Best of asset or cash" & $\max \left(S_{1}, S_{2}, \ldots, S_{n}, K\right)$ \\
"Call on max" & $\max \left(\max \left(S_{1}, S_{2}, \ldots, S_{n}\right)-K, 0\right)$ \\
"Put on min" & $\max \left(K-\min \left(S_{1}, S_{2}, \ldots, S_{n}\right), 0\right)$ \\
"Put 2 and Call 1" & $\max \left(S_{1}-S_{2}, 0\right)$ \\
"Multi-strike" & $\max \left(S_{1}-K_{1}, S_{2}-K_{2}, \ldots, S_{n}-K_{n}, 0\right)$ \\
"Pyramid" & $\max \left(\left|S_{1}-K_{1}\right|+\left|S_{2}-K_{2}\right|+\ldots+\left|S_{n}-K_{n}\right|-K, 0\right)$ \\
"Madonna" & $\max \left(\sqrt{\left(S_{1}-K_{1}\right)^{2}+\ldots+\left(S_{n}-K_{n}\right)^{2}}-K, 0\right)$ \\
\hline
\end{tabular}

For these options, one can apply the approach described above. Of course, there are options whose functions are neither convex nor concave. These can be easily obtained by taking combinations of long and short positions on the European call and put options with the same exercise time, for example, a collar. Binary options provide another example of such options, moreover, with a discontinuous payoff function. Examples of rainbow options with payoff functions that are not convex are the European rainbow options in Table 2. 
Table 2. Examples of European rainbow options with non-convex payoff functions.

\begin{tabular}{ll}
\hline Option Type & Payoff Function \\
\hline "Call on $\min "$ & $\max \left(\min \left(S_{1}, S_{2}, \ldots, S_{n}\right)-K, 0\right)$ \\
"Put on $\max ^{\prime}$ & $\max \left(K-\max \left(S_{1}, S_{2}, \ldots, S_{n}\right), 0\right)$ \\
\hline
\end{tabular}

\section{Conclusions}

If the payoff functions are convex and the compacts describing possible increments of discounted prices are convex, the solutions of Bellman-Isaacs equations are also convex.

Under mild conditions, a game equilibrium holds, as well as the upper semicontinuity of the multivalued mapping, that associates to each price history the set of corresponding optimal mixed strategies of the market concentrated in at most $n+1$ extreme point ( $n$ being the number of risky assets). These optimal mixed strategies and their supports can display regular behavior upon assuming some additional conditions of geometrical type; we illustrate this behavior using a two-dimensional market model of a multiplicative form with no trading constraints as an example.

In the present paper, we do not address model calibration issues (for a recent overview, see Reference [41] for a study focused on calibrating a model with real market data), but this question, which is important in practice, will be a part of our future research, together with a study of model sensitivity and relevant numerical methods.

Funding: Support from the Basic Research Program of the National Research University Higher School of Economics is gratefully acknowledged.

Conflicts of Interest: The author declares no conflict of interest.

\section{References}

1. Smirnov, S.N. Guaranteed deterministic approach to superhedging: Market model, trading constraints and Bellman-Isaacs equations. Math. Games Theory Appl. 2018, 10, 59-99.

2. Smirnov, S.N. Guaranteed deterministic approach to superhedging: "No arbitrage" properties of the market. Math. Games Theory Appl. 2019, 11, 68-95.

3. Smirnov, S.N. Guaranteed deterministic approach to superhedging: Properties of semicontinuity and continuity of solutions of Bellman-Isaacs equations. Math. Games Theory Appl. 2019, in print.

4. Smirnov, S.N. A Guaranteed Deterministic Approach to Superhedging: Lipschitz Properties of Solutions of the Bellman-Isaacs Equations. In Frontiers of Dynamics Games. Game Theory and Management, St. Petersburg, 2018; Petrosyan, L.A., Mazalov, V.V., Zenkevich, N.A., Eds.; Birkhäuser: Basel, Switzerland, 2019; pp. 267-288.

5. Smirnov, S.N. Guaranteed deterministic approach to superhedging: Mixed strategies and game equilibrium. Math. Games Theory Appl. 2019, under review.

6. Smirnov, S.N. Guaranteed deterministic approach to superhedging: Equilibrium in the case of no trading constraints. J. Math. Sci. 2020, 248, 105-115.

7. Smirnov, S.N. Guaranteed deterministic approach to superhedging: Most unfavorable market scenarios and the problem of moments. Math. Games Theory Appl. 2019, submitted.

8. Smirnov, S.N. Guaranteed deterministic approach to superhedging: Properties of optimal "market" strategies and their supports. Math. Games Theory Appl. 2019, submitted.

9. Föllmer. H.; Schied, A. Stochastic Finance. An Introduction in Discrete Time, 4th ed.; Walter de Gruyter: New York, NY, USA, 2016.

10. Knight, F.H. Risk, Uncertainty and Profit; Houghton Mifflin Co.: New York, NY, USA, 1921.

11. Andreev, N.A.; Smirnov, S.N. Guaranteed approach in investment and hedging. In «Tikhonovskie Chteniya»: Scientistic Conference Proceedings: Dedicated to Academic Andrey Nikolaevich Tikhonov: 19 October-2 November 2018; MAKS Press: Moscow, Russia, 2018; p. 11.

12. Carassus, L.; Gobet, E.; Temam, E. A class of financial products and models where super-replication prices are explicit. The 6th the Ritsumeikan International Conference on Stochastic processes and applications to mathematical finance. In Stochastic Processes and Applications to Mathematical Finance; Akahori, J., Ogawa, S., Watanabe, S., Eds.; World Scientific: Singapore, 2006; pp. 67-84 
13. Carassus, L.; Vargiolu, T. Super-Replication Price for Asset Prices Having Bounded Increments in Discrete Time. 2010. Available online: https://hal.archives-ouvertes.fr/hal-00511665/ (accessed on 10 December 2019).

14. Kolokoltsov, V.N. Nonexpansive maps and option pricing theory. Kybernetika 1998, 34, 713-724.

15. Dana, R.-A.; Le Van, C. On the different notions of arbitrage and existence of equilibrium. J. Econ. Theory 1999, 87, 169-193. [CrossRef]

16. Bernhard, P.; Engwerda, J.C.; Roorda, B.; Schumacher, J.; Kolokoltsov, V.; Saint-Pierre, P.; Aubin, J.-P. The Interval Market Model in Mathematical Finance: Game-Theoretic Methods; Springer: New York, NY, USA, 2013.

17. Smirnov, S.N. Thoughts on Financial Risk Modeling: The Role of Interpretation. Intell. Risk 2012, 2, 12-15.

18. Hobson, D. Robust hedging of the lookback option. Financ. Stoch. 1998, 2, 329-347. [CrossRef]

19. Obloj, J.; Wiesel, J. A unified Framework for Robust Modelling of Financial Markets in Discrete Time. arXiv 2019, arXiv:1808.06430.

20. Bouchard, B.; Nutz, M. Arbitrage and duality in nondominated discrete-time models. Ann. Appl. Probab. 2015, 25, 823-859. [CrossRef]

21. Bayraktar, E.; Zhou, Z. On arbitrage and duality under model uncertainty and portfolio constraints. Math. Financ. 2017, 27, 988-1012. [CrossRef]

22. Bayraktar, E.; Zhang, Y. Fundamental theorem of asset pricing under transaction costs and model uncertainty. Math. Oper. Res. 2016, 41, 1039-1054. [CrossRef]

23. Burzoni, M.; Frittelli, M.; Hou, Z.; Maggis, M.; Obloj, J. Pointwise arbitrage pricing theory in discrete time. Math. Oper. Res. 2019.10.1080/01932691.2010.513279. [CrossRef]

24. Burzoni, M.; Frittelli,M.; Hou, Z.; Maggis, M. Universal arbitrage aggregator in discrete-time markets under uncertainty. Financ. Stoch. 2016, 20, 1-50. [CrossRef]

25. Burzoni, M.; Frittelli, M.; Maggis, M. Model-free superhedging duality. Ann. Appl. Probab. 2017, 27, 1452-1477. [CrossRef]

26. Aksamit, A.; Deng, S.; Obloj, J.; Tan, X. Robust pricing-hedging duality for American options in discrete time financial markets. Math. Financ. 2019, 29 861-897. [CrossRef]

27. Bergman, Y.Z.; Grundy, B.D.; Wiener, Z. General Properties of Option Prices. J. Financ. 1996, 51, $1573-1610$. [CrossRef]

28. Leichtweiß, K. Konvexe Mengen; Springer: Berlin, Germany, 1979.

29. Rockafellar, R.T. Convex Analysis; Princeton University Press: Princeton, NJ, USA, 1970.

30. Polovinkin, E.S. Multi-Valued Analysis and Differential Inclusions; Fizmatlit: Moscow, Russia, 2015.

31. Hu, S.; Papageorgiou, N. Handbook of Multivalued Analysis, Volume I: Theory; Springer: Berlin, Germany, 1997.

32. Kupka, I. Continuous selections from topological to metric spaces. Rend. Circ. Mat. Palermo 1990, 39, $427-435$. [CrossRef]

33. Edmonds, A.L. Simplicial decompositions of convex polytopes. Pi Mu Epsil. J. 1970, 5, 124-128.

34. Simchi-Levi, D.; Chen, X.; Bramel, J. The Logic of Logistics: Theory, Algorithms, and Applications for Logistics Management; Springer: New York, NY, USA, 2013.

35. Birkhoff, G. Latice Theory, 3rd ed.; American Mathematical Society: Providence, RI, USA, 1967.

36. Steinitz, E. Bedingt konvergente Reihen und konvexe Systeme (Fortsetzung). J. Reine Angew. Math. 1914, 144, $1-40$.

37. Hucki, Z.; Kolokoltsov, V.N. Pricing of rainbow options: Game theoretic approach. Int. Game Theory Rev. 2007, 9, 215-242. [CrossRef]

38. Dana, R.-A.; Jeanblanc-Picqué, M. Marchés Financiers en Temps Continu, 1st ed.; Economica: Paris, France, 1994.

39. Cox, J.C.; Ross, S.A.; Rubinstein, M. Option pricing: A simplified approach. J. Financ. Econ. 1979, 7, $229-263$. [CrossRef]

40. Vinogradov, I.M. Basics of Number Theory; Nauka: Moscow, Russia, 1972.

41. Singh, S.; Bhardwaj, N.; Sharma, G.; Kaya, T.; Mahendru, M.; Erkut, B. Research in market-calibrated option pricing analysis: A systematic review and research agenda. In Qualitative Research in Financial Markets; Emerald Publishing Limited: Bingley, UK, 2019; doi:10.1108/QRFM-01-2019-0004. [CrossRef] 